\title{
Politiques urbaines et ateliers de fabrication numérique (AFN) : fabriquer en ville pour fabriquer la ville - le cas de Barcelone
}

\author{
Jérémy Diaz ${ }^{a}$, Sylvain Lefebvre ${ }^{b}$
}

RÉSUMÉ. La diffusion des ateliers de fabrication numérique (AFN) est devenue un phénomène mondialisé. Principalement localisés en milieu urbain, ces ateliers ont la volonté de mettre à la disposition des citadins des moyens de production afin de concevoir, de réparer et de prototyper toutes sortes d'objets. Depuis quelques années, nous avons constaté un enthousiasme croissant des pouvoirs urbains pour ces pratiques popularisées par le mouvement maker. L'objectif de cet article est de comprendre les relations qu'entretient ce phénomène avec les gouvernements urbains. Peu de choses ont été écrites sur la façon dont les villes mettent en œuvre des stratégies pour encourager ces pratiques sur leur territoire. Pour combler cette lacune, nous avons analysé le cas de la ville de Barcelone, qui mène une action territoriale ambitieuse à travers la création d'un réseau public d'ateliers dédiés à la fabrication numérique.

\begin{abstract}
The spread of makerspaces has become a global phenomenon. Mainly located in urban areas, these spaces provide urban dwellers with means of producing, designing, repairing and prototyping all kinds of objects. Since a few years, we have seen growing enthusiasm with urban powers for the maker movement practices. The objective of this article is to help understand the relationships between this phenomenon and urban institutions. Little has been written on how cities are implementing strategies to promote these practices. Thus, we here analyze the case of the city of Barcelona that is conducting an ambitious territorial operation by creating a public network of places dedicated to digital fabrication.
\end{abstract}

\section{Introduction}

Les pouvoirs publics s'intéressent de plus en plus aux pratiques collaboratives entourant la fabrication d'objets. Médiatisées par le mouvement ma$k e r^{1}$, ces pratiques se sont progressivement diffusées depuis 15 ans à travers l'organisation d'événements et la multiplication internationale de divers ateliers de fabrication numérique (AFN). Quelle que soit leur appellation, ces AFN ont en commun d'encourager l'expérimentation et la créativité, de faciliter l'apprentissage et la maittrise technique d'outils numériques, et de valoriser l'entraide en offrant un accès compétitif à des moyens de production traditionnellement réservés à des professionnels spécialisés dans la conception et le prototypage d'objets. Alors que l'apport de financement public apparaissait comme une incongruité pour les premiers ateliers militants, soucieux de conserver un esprit libertaire et une autonomie complète dans la nature de leurs activités, la situation a bien évolué. Désormais, les pouvoirs publics investissent de plus en plus dans la promotion et la diffusion territoriale des $\mathrm{AFN}$, ces derniers étant perçus comme des leviers dynamiques de développement socioéconomique. Ils les intègrent dans des cadres d'action plus larges et diversifiés, en risquant d'édulcorer l'essence subversive des premières initiatives qui a fait le succès du mouvement.

La révolution numérique a augmenté de façon considérable les voies d'accès et de création du savoir par le grand public. La multiplication des flux d'information bouleverse nos modes d'appropriation

\footnotetext{
a Doctorant, Département d'étude urbaines, École des sciences de la gestion, Université du Québec à Montréal

b Professeur titulaire, Ph. D., Département de géographie, Université du Québec à Montréal
} 
du savoir en général. Plus précisément, un tel bouleversement oblige les pouvoirs publics à repenser l'acte d'apprendre en adaptant les politiques éducatives territoriales. Le succès renouvelé du bricolage, du démontage, de la réparation, du codage et de la sophistication au sein d'une nouvelle vague d'ateliers équipés de technologies a été rapidement identifié par quelques gestionnaires urbains comme une occasion de valoriser la culture scientifique, technique et industrielle au sein de la population. Il s'agit de créer des vocations, d'encourager l'innovation et de préparer le citoyen de demain à vivre dans un environnement urbain qui contiendra de multiples dispositifs technologiques.

Se pose ainsi un défi d'autonomie et d'émancipation collective que les promoteurs du mouvement maker promettent de relever en favorisant la " démocratisation » des processus de conception et de fabrication numériques. Pourtant, en visitant de nombreux AFN, et ce, dans diverses villes d'Amérique du Nord et d'Europe, nous avons pu observer certaines barrières à l'accès aux ressources technologiques et une forme d'homogénéité socioéconomique parmi les participants. À rebours des discours sur l'ouverture à tous et sur la démocratisation de ces savoirs, les ateliers d'expérimentation portés par ces acteurs présentent souvent des formes de sélection des usagers et des bénéficiaires des projets qui interpellent et remettent en question l'esprit même du mouvement maker.

C'est pourquoi il est légitime de se demander si certains AFN sont plus souhaitables que d'autres pour tendre vers cet objectif d'éducation populaire et d'appropriation sociale de ces lieux. Plus précisément, nous allons explorer le cas d'un réseau d'ateliers publics à Barcelone en décrivant la façon dont ce service souhaite créer les conditions d'une réelle appropriation citoyenne des technologies de fabrication numériques, alors que la structure porteuse - en l'occurrence la ville de Barcelone est régie par des pratiques institutionnelles normées et très encadrées.

\section{Typologie des AFN}

La fabrication numérique requiert des compétences et de l'expérience avant de parvenir à des résultats satisfaisants. La capacité pour un individu de fabriquer un objet à partir d'une idée, d'un dessin ou d'un besoin nécessite le développement de connaissances théoriques et pratiques telles que la formulation d'un projet, l'énumération de problèmes techniques, la recherche d'une solution technique viable, la maitrise des logiciels de modélisation et de fabrication numérique ou encore la capacité d'utiliser de manière autonome des machines de fabrication numérique. Dans le domaine de l'éducation, le mouvement des makers a encouragé l'aménagement d'ateliers flexibles d'apprentissage (Halverson et Sheridan, 2014).

Le maker est une sorte de bricoleur du XXIe siècle. Il fait un usage important du réseau Internet comme lieu de diffusion de connaissances, de collaborations et de communications. Il accède à des outils complexes à bas coût autrefois réservés aux entreprises de pointe. La créativité, la bidouille, la fabrication d'objets par soi-même (DIY - do it yourself) et l'expérimentation sont pour lui autant de moyens d'agir sur le monde et sur sa vie. Il revendique le droit de comprendre et d'intervenir techniquement sur les choses qu'il utilise quotidiennement comme condition de sa liberté (Fourmond et Gastall, 2014, s. p.).

L'identité ambiguë du maker a évolué au fil du temps : elle désigne à la fois un activiste, un passionné de bricolage ou d'électronique, voire un nouveau type d'entrepreneur. Dans la francophonie, le terme atelier de fabrication numérique (AFN) est davantage répandu pour désigner les makerspaces. Ces ateliers éphémères ou permanents promeuvent un apprentissage par la pratique (learning by doing) dans un cadre social favorisant le travail d'équipe. En effet, les AFN encouragent les rencontres, les échanges et les collaborations en personne et en ligne. Comme le décrivent Sheridan et ses collaborateurs (2014), la participation à ces ateliers permet, entre autres, de manipuler divers outils, de résoudre des problèmes techniques, de réaliser des croquis, de s'engager comme membre au sein de collectifs, d'assumer des rôles de leadership et d'enseignement au besoin et, enfin, de partager ses créations et ses compétences avec un grand nombre de personnes.

Le nombre d'AFN s'est accru de manière significative depuis la crise économique de 2008. Une récente enquête révélait que plus de $26 \%$ des villes 
américaines disposaient d'au moins un atelier mutualisant des outils et machines de fabrication (National League of Cities, 2016). En Europe, une autre étude a recensé 826 ateliers équipés d'outils techniques au sein des États de l'Union : $41 \%$ de ces ateliers étaient situés en France, en Allemagne ou en Italie (Rosa, Ferretti, Panella et Wanner, 2017). En dépit des diverses appellations (makerspaces, hackerspaces, hacklabs, fab labs, etc.), nous définissons de manière générique les AFN comme des ateliers ouverts au public mettant à leur disposition des machines-outils à commande numérique, mais aussi l'univers numérique d'information et de communication qui en émane (sites Internet, conversations numériques, bases de données, etc.). Ces ateliers rassemblent des participants autour de la pratique du faire qui génèrent des formes d'interactions et de sociabilités dérivées de la culture numérique. Ce sont également des sites de production de contenu matériel et immatériel dont l'ancrage territorial peut aussi bien être physique que virtuel, du fait de la nature des moyens de production.

Historiquement, l'existence d'ateliers techniques ouverts au grand public n'est pas nouvelle. En 1821, les mechanics' institutes créés à Édimbourg offraient un enseignement technique à la classe ouvrière (Kelly, 1952). En 1847, le socialiste Louis Blanc défendait la création d'ateliers sociaux en France (Demoustier et Rousselière, 2005). Plus près de nous, les cybercafés ont favorisé l'apprentissage et l'utilisation des premiers ordinateurs personnels (Lægran et Stewart, 2003). L'originalité des AFN émane de la combinaison de deux dimensions auparavant séparées pour le grand public : la fabrication d'objets et l'informatique. Pour cette raison, ces ateliers peuvent également se définir comme des sites de conception et de production connectés et organisés en réseaux.

Les contours de ces ateliers sont toutefois difficiles à cerner (Eychenne, 2012; Smith, Hielscher, Dickel, Soderberg et van Oost, 2013; Capdevila, 2017; Pacchi, 2017)2 ${ }^{2}$. Ils mettent en évidence des formes variables de partage, d'occupation, d'aménagement et d'appropriation. Les structures porteuses ainsi que le rapport à l'espace et au territoire qu'entretiennent les usagers de ces ateliers diffèrent. En ce sens, ces ateliers contribuent à une différenciation des projets et de leur milieu d'insertion. Ils partici-

Revue Organisations E Territoires • Volume $28 \cdot \mathrm{N}^{\circ}{ }_{2} \cdot 2019$ pent donc à la production d'une géographie distincte. Nous proposons de classer ces ateliers de fabrication numérique (AFN) en six catégories.

\section{1) Les $A F N$ communautaires}

Créés par une communauté de militants passionnés d'informatique, d'électronique et des technologies de fabrication (voir photo 1), ces ateliers fonctionnent par un mode de régulation autonome et ahiérarchique inspiré des communautés numériques et respectant dans une certaine mesure l'éthique des hackers (Levy, 2010). Certains évoquent la « do-ocratie » (le pouvoir par le faire, to do) comme mode d'organisation de ces ateliers: les individus s'assignent eux-mêmes des tâches et les exécutent (Lallement, 2015). Le pouvoir et l'autorité des individus y sont proportionnels aux tâches qu'ils ont choisi d'accomplir. Cependant, des barrières d'accès aux machines et aux ateliers ont été identifiées ainsi que des mécanismes d'entre-soi, voire une homogénéité sociale et culturelle des membres de la communauté (Ferchaud, 2017).

\section{2) Les AFN publics}

Créés par les pouvoirs publics au sein d'écoles, de bibliothèques, de musées ou de locaux communautaires, ces ateliers ont pour mission d'offrir un service d'éducation et de médiation culturelle aux sciences et aux technologies à travers la conception et la fabrication d'objets. Ils offrent des cours, des formations adaptées ou des animations ouvertes au grand public. Le fonctionnement de ces ateliers est régi par les principes et les valeurs d'un service public, à savoir une égalité de traitement, la continuité et la gratuité (variable) du service.

\section{3) Les AFN universitaires}

Créés par une université, ces ateliers sont réservés aux étudiants et au personnel enseignant pour apprendre de nouvelles choses, travailler avec leurs pairs, envisager de nouvelles idées, explorer, bricoler, inventer et fabriquer (Harris et Cooper, 2015). Ces ateliers sont régis par les normes et les règles de l'établissement. Le personnel peut être composé d'étudiants et de personnel spécialisé (Barrett et collab., 2015). Les activités de ces ateliers vont du projet pluridisciplinaire de recherche à des ateliers de formation, en passant par des séances réservées à la préparation de cours ou de projets personnels (Wong et Partridge, 2016). 


\section{4) Les AFN d'entreprise}

Créés par les entreprises, ces ateliers sont conçus comme des espaces de travail collaboratif pour les salariés, les collaborateurs et les partenaires. Ils visent à impulser des dynamiques d'innovation ascendante (bottom-up) à travers des pratiques exploratoires centrées sur le bricolage, l'improvisation, le prototypage et la conception innovante (Lô, 2017). Remplaçant ou complémentant les départements de recherche et de développement coûteux, ces ateliers offrent des formations et de l'accompagnement aux porteurs de projets à l'interne. Ils seraient donc propices au développement individuel et organisationnel des salariés, c'est-à-dire non seulement à acquérir des compétences, mais aussi à exploiter commercialement les projets mis en œuvre.

\section{5) Les AFN commerciaux}

Créés par des entrepreneurs, ces ateliers combinent plusieurs types d'activités, dont un service de fabrication numérique. Majoritairement, ce sont des cafés (voir photo 2) ou des espaces de cotravail qui ont ouvert en leur sein des ateliers dédiés à la conception, à la fabrication et au prototypage pour encourager des liens et des synergies entre ces différentes activités. Ouverts à tous ou réservés aux coworkers, ces ateliers vendent des services pouvant être prohibitifs pour le grand public, mais répondent à un besoin de plusieurs catégories professionnelles, par exemple les designers, les architectes ou les ingénieurs.

\section{6) Les AFN industriels}

Nommés en anglais small-scale manufacturing spaces, ces ateliers désignent les espaces de grande superficie qui regroupent des autoentrepreneurs ou de petites entreprises intéressées par la production de biens matériels nécessitant des outils et des machines industrielles (voir photo 3). Ces ateliers offrent également des bureaux, des formations et des services d'incubation et d'accompagnement pour le prototypage et la production à petite échelle. L'accès est payant et le public visé est composé de professionnels dans le domaine manufacturier.

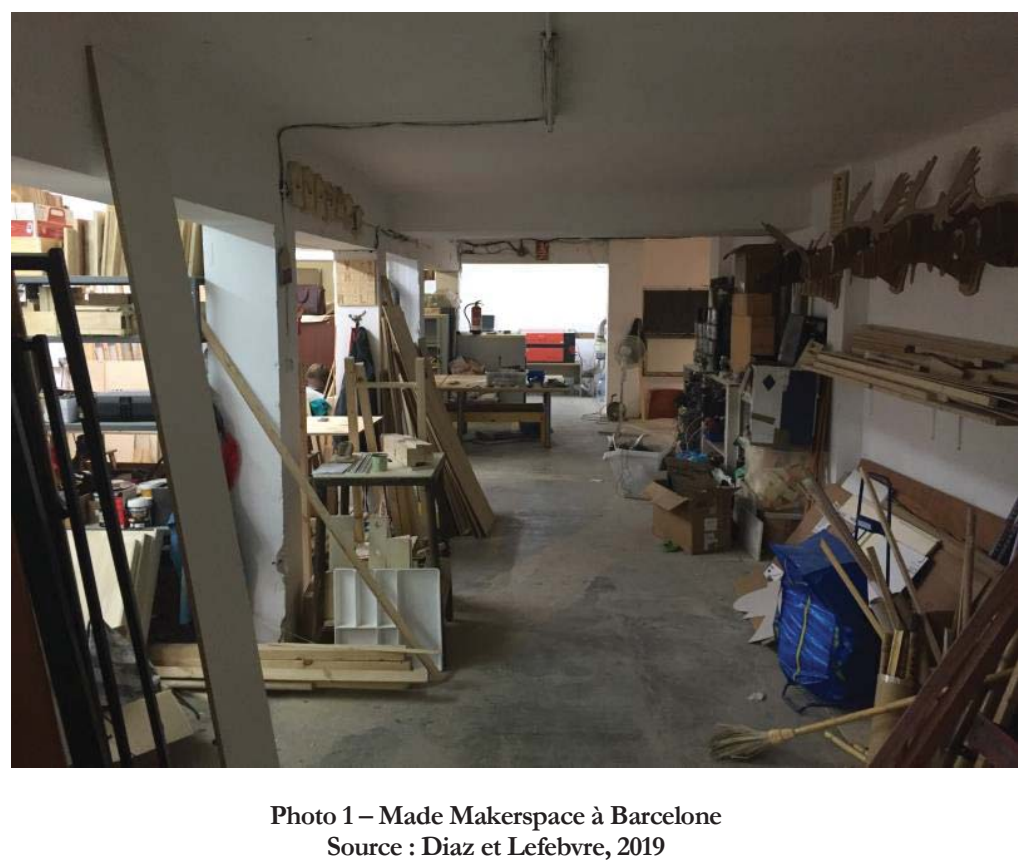




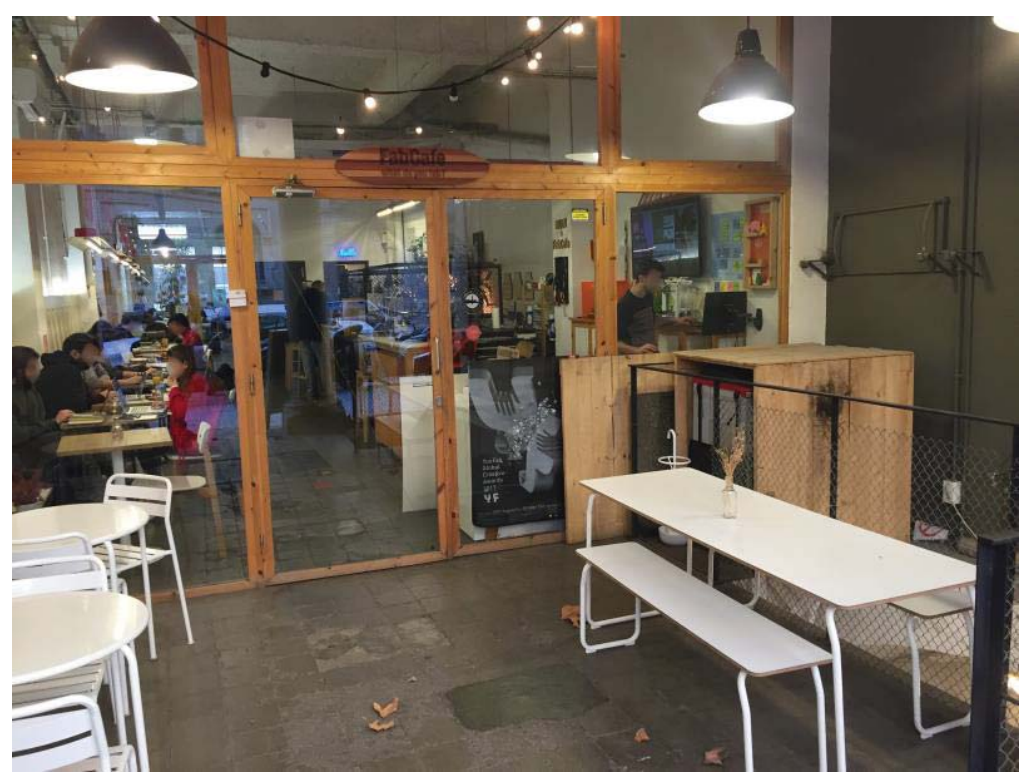

Photo 2 - Fab Café à Barcelone

Source : Diaz et Lefebvre, 2019

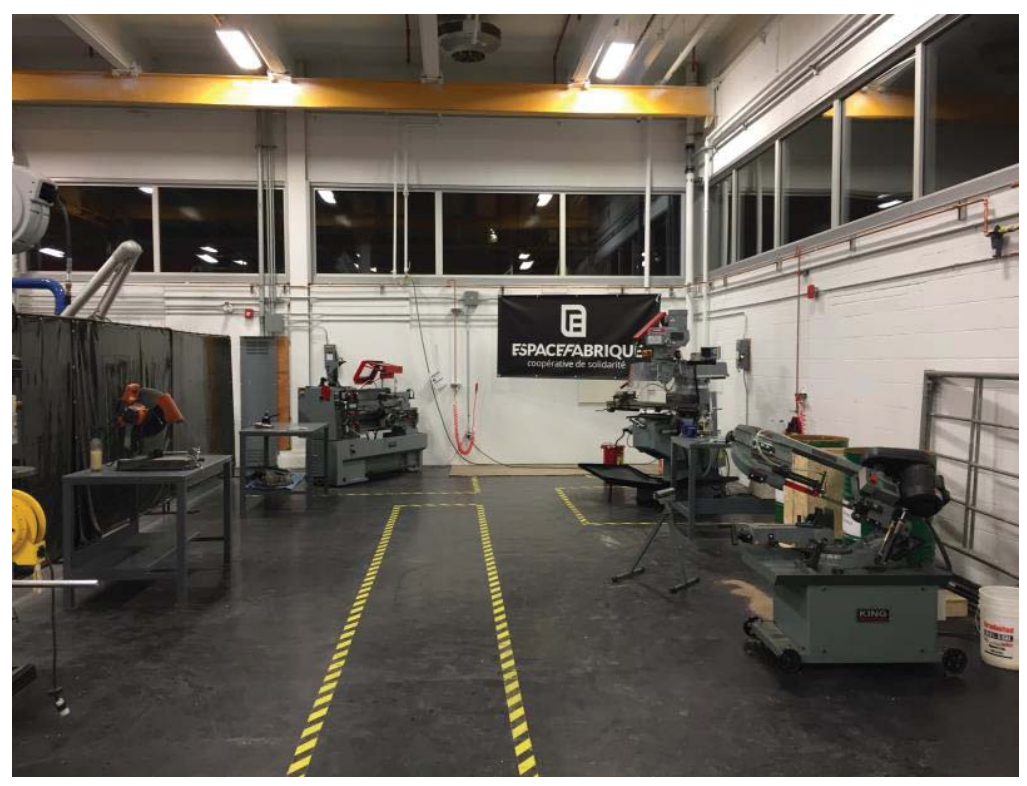

Photo 3 - Espace Fabrique à Montréal Source : Diaz et Lefebvre, 2019

Cette typologie dessine une géographie des AFN. De plus en plus, ils sont devenus des outils de développement territorial (Liefooghe, 2018). Le développement territorial se fonde sur l'idée selon laquelle un territoire doit son développement à l'organisation et à l'implication d'acteurs localisés qui se reconnaissent dans des projets communs (Torre, 2015; Pecqueur, 2016). Ces territoires se construisent en constituant des capacités collectives de production, en formant des dynamiques locales spontanées ou en créant des projets mobilisateurs portés par des leaders (Klein,
2016). Ces dynamiques territoriales reposent le plus souvent sur le partage de connaissances et de savoirfaire entre acteurs, ce qui stimule des formes d'innovations sociales et technologiques (Torre, 2015; Klein, Soussi, Koop et Pecqueur, 2016). Ainsi, les ateliers de fabrication numérique, comme d'autres ateliers hybrides émergents, peuvent être identifiés comme des dispositifs d'innovation territoriale (Klein et Pecqueur, 2017) dont se saisissent majoritairement les villes. 


\section{Politiques urbaines autour des AFN}

Depuis quelques années, nous avons constaté un enthousiasme croissant des pouvoirs publics pour promouvoir ces pratiques en les articulant à des plans d'action plus larges grâce à des subventions publiques, à l'organisation d'événements ou encore à des projets de régénération urbaine de quartiers dévitalisés. La localisation des AFN se situe principalement en milieu urbain, particulièrement dans les métropoles. À Paris, on n'en recense pas moins d'une centaine (APUR, 2016). Il est donc important de clarifier ici les raisons qui poussent les pouvoirs publics à organiser, à renforcer et à réguler la croissance de ces pratiques au sein d'une diversité d'ateliers dans la ville.

\subsection{La déclinaison des politiques nationales éducatives}

À l'échelle nationale, l'intérêt des pouvoirs publics pour le mouvement maker s'explique par l'enjeu stratégique que représentent la formation, l'attraction et la rétention d'une main-d'œuvre hautement qualifiée dans un contexte économique globalisé et concurrentiel. Chaque décennie, un nouvel ensemble de compétences et d'activités intellectuelles devient crucial pour le travail, la vie en société et la citoyenneté. Au début des années 1970, la programmation informatique était une de ces compétences fondamentales, mais les ordinateurs d'alors étaient des machines inabordables et, par conséquent, l'utilisation en classe était inconcevable (Blikstein, 2013). La baisse du coût des machines à commande numérique dans les années 2000 entrouvre alors une nouvelle étape dans l'intégration des technologies en éducation. À l'instar des Etats-Unis, plusieurs pays ont mis en œuvre des politiques publiques pour restructurer les programmes scolaires, pour renouveler l'éducation informelle et parascolaire ainsi que pour encourager la formation continue.

Dès 2009, l'administration américaine Obama a annoncé l'objectif que les États-Unis deviennent une nation de makers en instaurant une politique d'aide aux ateliers de fabrication collaboratif, à l'éducation transdisciplinaire STEAM (science, technology, engineering, arts and maths) et aux entreprises en démarrage (start-ups). À titre d'exemple, cette vision s'est traduite par la création d'un fonds californien de 17,5 M\$ US en 2016 pour subventionner l'ouverture d'ateliers collaboratifs dans chaque établissement scolaire (California Community College, 2016).

Cette politique d'aide directe a été suivie par le gouvernement britannique dans son Plan de stratégie numérique. Concrètement, la reconnaissance institutionnelle des retombées éducatives de ces ateliers s'est matérialisée par la publication d'un rapport commandé par le British Council intitulé Libraries deliver: Ambition for public libraries in England 2016 to 2021 (Government of the United Kingdom, 2016). Ce rapport soutient l'implantation d'ateliers collaboratifs au sein des bibliothèques publiques pour aider les Britanniques à acquérir des compétences numériques.

L'État français, pour sa part, a soutenu financièrement 14 AFN avec une enveloppe budgétaire de 2,2 M€ en 2012. Le gouvernement français prévoit d'engager, à hauteur de $110 \mathrm{M} €$ sur trois ans (20202022), un fonds d'aide à la création et à la pérennisation de 300 «fabriques de territoires» appelées tiers-lieux, dont plusieurs renfermeront des AFN, ainsi que la mise en place d'une structure nationale pour structurer le réseau (CGET, 2018).

Ces relations ne se réduisent pas aux pays occidentaux. Des exemples similaires sont identifiables dans le domaine de l'éducation en Chine et en Afrique du Sud (Wen, 2017).

\subsection{L'instauration de partenariats public-privé}

Les administrations municipales ne possèdent pas les compétences techniques ni humaines pour assurer le fonctionnement de ces ateliers. La plupart des villes ont recours à des partenariats public-privé pour financer et gérer ces équipements. Ce mode de financement permet à l'autorité publique de faire appel à des prestataires privés pour assurer un service public en contrepartie d'un paiement public à l'organisme gestionnaire. Dans d'autres cas observés, les villes accordent une aide financière à une entreprise privée par l'octroi de subventions ou par le prêt d'un local à prix modique.

Au tournant des années 2000, la baisse du coût des machines à commande numérique et des logiciels de conception a permis à de nouveaux acteurs d'offrir ces services en répondant à des appels d'offres publics. L'appartenance à des réseaux globaux tels que le mouvement maker ou les fab labs de la part de 
certains soumissionnaires était promue comme un actif stratégique dans la crédibilité de leur offre.

\subsection{La stimulation de l'entrepreneuriat et de l'innovation}

La référence aux AFN se multiplie dans les plans d'action économique de plusieurs villes nordaméricaines. La littérature s'accorde pour dire que ces ateliers ont le potentiel de stimuler l'entrepreneuriat local en abaissant les coûts de formation, de prototypage, d'assistance technique et de commercialisation d'un produit (Hagel, Brown et Kulasooriya, 2014; Wolf-Powers et collab., 2017). Ils généreraient des opportunités en matière d'entrepreneuriat local, une diversification de la maind'œuvre ainsi qu'une aide à la coordination et à l'animation de réseaux socioéconomiques au sein des territoires sur lesquels ils se situent (Suire, 2016; Liefooghe, 2017; Mariotti, Pacchi et Di Vita, 2017).

À titre d'exemple, l'ancienne ville industrielle de Pittsburgh a mis en œuvre un plan d'innovation inclusive (2015-2018), dont l'objectif est de « relier le fossé numérique et de créer des occasions pour les habitants de Pittsburgh de participer à la nouvelle économie. Viser l'inclusion signifie de fournir des occasions pour l'économie de l'innovation en haute technologie et en compétences élevées » (URAP, 2015, p. 4, trad. libre). Parmi les actions proposées, le renforcement des collaborations avec les organisations makers et les espaces de cotravail est présenté comme essentiel au développement d'un écosystème de structures d'accompagnement pour inciter, former et aider les entrepreneurs dans les premières phases de croissance.

Le Département du développement économique de Calgary a, pour sa part, publié un rapport intitulé How makerspaces support innovative urban economies (CED, 2017), qui vise à former et à retenir les entrepreneurs, à exploiter les atouts industriels et technologiques du tissu économique local, et à aider à remédier à la vacance de l'immobilier commercial de la ville. Dans ce document, les ateliers collaboratifs sont appréhendés comme des ateliers capables de prototyper non seulement de nouveaux biens, mais également de faciliter l'accès à des savoirs stratégiques pour les acteurs économiques locaux, tels que l'état d'un marché potentiel, les dernières innovations, les montages financiers collaboratifs ou la gestion de projet.

\subsection{L'animation culturelle : musées et festivals}

La révolution numérique a accru les possibilités offertes au grand public d'accéder à des contenus scientifiques, technologiques ou artistiques. Non seulement les voies d'accès au savoir se sont multipliées avec Internet (sites de vulgarisation, réseaux sociaux), mais également les opportunités d'en créer (Wikipédia, DIY, biologie de garage). Cela oblige les pouvoirs publics à renouveler leur programmation culturelle en adoptant de nouvelles approches qui se démarquent des initiatives traditionnelles. C'est la raison pour laquelle de nombreux musées ont implanté de tels ateliers en leur sein (Brahms et Crowley, 2016).

Par ailleurs, il existe plusieurs exemples de villes qui subventionnent l'organisation d'événements en lien avec ces pratiques. À petite échelle, la Ville d'Atlanta, en Géorgie, a subventionné des camps d'été pour aider les AFN à générer des revenus stables de leurs activités (Van Holm, 2015). D'autres festivals peuvent regrouper plusieurs milliers de participants autour d'ateliers, de conférences ou d'expositions, par exemple les Maker Faires, les Manufacturing Days ou, plus traditionnellement, les marchés de Noël. Ces événements commencent à se diversifier: la Ville de Calgary organise un festival maker autour de la conception de technologie prête-à-porter (wearable technology).

\subsection{La réglementation et les pratiques urbanistiques}

Devant le foisonnement des initiatives, les administrations municipales commencent à réfléchir sur la nécessité d'encourager ou de réguler le déploiement de ces ateliers à travers l'adaptation des formes d'occupation du sol, le code de la construction ou encore l'application des normes de sécurité.

À propos de Toronto, Bowden (2016) relate les difficultés auxquelles certains ateliers communautaires font face en matière d'inspection des bâtiments. L'injonction de mettre aux normes ces locaux - souvent loués à bas prix - déstabiliserait la pérennité de ces communautés. Paradoxalement, la reconnaissance institutionnelle de ces ateliers participe à réduire le spectre de leurs activités. L'application légale des normes sur le bruit, l'évacuation des vapeurs et des fumées, la nature des outils et machines utilisables ou 
encore la configuration spatiale permettraient de réguler indirectement leur capacité de production. L'esthétique d'un atelier, avec l'absence visible de certaines contraintes, participe souvent à faire de celui-ci un espace d'expérimentation.

Ces ateliers ont pourtant été identifiés comme des leviers viables de réhabilitation de locaux vacants dans d'anciens quartiers industriels. Un récent rapport sur les ateliers industriels indique que la question de l'occupation du sol constitue un enjeu central pour l'émergence de ces ateliers, dans un contexte où le zonage commercial a tendance à s'étendre aux anciens quartiers industriels (Smart Growth America, 2017). Pour conserver une activité industrielle dans un de ses quartiers, la Ville de San Francisco autorise la construction de bureaux sur des terrains industriels uniquement si l'opération s'accompagne de l'implantation d'activités industrielles légères (Pratt Center, 2016). D'autres villes ont adapté leur réglementation pour l'implantation des ateliers. La Ville d'Ottawa (2018) s'est récemment engagée à étendre son zonage industriel-commercial mixte à cet effet, tandis que le Département de la planification urbaine de New York a entamé une réflexion à l'échelle des anciens bâtiments industriels pour encourager des usages mixtes (NYC Planning, 2018). Ces ateliers y sont appréhendés comme des solutions immobilières susceptibles de combler la baisse des demandes pour des locaux commerciaux. Combinant un site de production en petite série et un espace de vente au détail en rez-dechaussée, ils représenteraient une option intéressante pour conserver des façades actives et pour promouvoir une ville à l'échelle humaine.

\subsection{L'appui à des foyers d'imaginaire urbain}

Dans les discours publics et les pratiques de la gestion urbaine, les édiles recourent de plus en plus à des modèles urbains conçus pour convaincre et guider l'action collective vers une évolution souhaitable de la ville (Wusten, 2016). Au cours des dernières décennies, l'accroissement de la mobilité internationale et des technologies de l'information et de la communication (TIC) ont accru le nombre et la circulation de ces modèles entre l'administration municipale et des communautés locales et internationales d'acteurs. Leur diffusion permet d'établir des comparaisons sur les façons de faire de différentes villes, de classifier les cas selon la qualité de réalisation du modèle, et d'identifier les conditions et bonnes pratiques qui facilitent le succès.

Dans cette perspective, les AFN s'apparentent à des composantes incontournables pour deux des référentiels urbains les plus répandus, à savoir la ville créative (Wen, 2017; Budge, 2018) ou la ville intelligente (Niaros, Kostakis et Drechsler, 2017). Les modèles dérivés de la Maker City (Hirshberg, Dougherty et Kadanoff, 2016) ou de la Fab City (Diez, 2018) sont en pleine expansion dans plusieurs parties du monde. L'apparition de ces modèles correspond à ces nouvelles formes de collaboration, dans un esprit de compétition entre les villes où les spécialistes du modèle en question dispensent leurs conseils aux communautés locales et à leurs représentants pour encourager l'adhésion à leur idéal, en vendant leurs services et compétences.

Depuis 2014, le défi des promoteurs de la Fab City de produire localement la majorité de ce que les villes consomment a réussi à convaincre 28 administrations municipales d'y participer. Ces promoteurs ont extrapolé le potentiel de fabriquer localement au sein des AFN vers l'idée d'une relocalisation mondiale de la production manufacturière et des échanges de biens physiques dans les métropoles. Cependant, les engagements sont variables et incitatifs. Il sera utile d'en comprendre les effets pratiques sur les politiques urbaines, car cet « effet de réseau » accroît la visibilité et l'acceptabilité sociale d'un modèle urbain dont les tenants et aboutissants restent à découvrir.

\section{Un projet pionnier à Barcelone : les ateneus de fabricació}

Depuis 2013, la ville de Barcelone met en oeuvre un réseau de lieux de fabrication numérique appelé Ateneus de Fabricació incarnant clairement la volonté de la ville d'institutionnaliser ces pratiques. Inspirés puis détournés du modèle des Fab Labs - ces espaces où il serait possible de tout fabriquer par les étudiants de l'université MIT -, les Ateneus de Fabricació offrent un service d'aide et d'accompagnement de création d'objets à l'ensemble des citoyens de la ville. À ce titre, le changement du nom du projet incarne la volonté de l'administration municipale d'inscrire cet ensemble de lieux dans un imaginaire urbain où les Ateneus représentaient des lieux destinés à l'éducation populaire de la classe ouvrière catalane à la fin du XIXe siècle. 
En ce sens, la mission des Ateneus de Fabricació est de « devenir un lieu où des personnes ayant des trajectoires, des antécédents, une formation et différentes compétences d'apprentissage peuvent expérimenter, créer ensemble, développer des activités et des projets liés à la fabrication numérique et s'impliquer dans l'ensemble du processus » (Ajuntament de Barcelona, 2016, p.5). Le développement de ce nouveau type d'espaces a nécessité une adaptation des modes de fonctionnement de l'administration publique qui ne bénéficiait pas de la compétence nécessaire en matière de compétence technique et de philosophie de gestion d'espaces où l'activité est grandement déterminée par la motivation et les projets initiés par les citoyens.

\subsection{L'enjeu de la gestion de ces ateliers}

Chaque ateneu de fabricació est actuellement géré par plusieurs organisations à but non lucratif choisies après des concours publics. Ces organisations paient le personnel, composé de trois profils distincts : 1) les technologues pour le soutien technique des projets, 2) un pédagogue pour l'animation de l'atelier et 3) la direction pour le bon fonctionnement du service et la coopération avec les organismes extérieurs. Les arrondissements de la ville (distritos) fournissent un local aménagé ainsi que les équipements technologiques (électronique, machines à commande numérique, outils, etc.) achetés par la Xarxa d'ateneus (XAF), la structure municipale composée d'un porteur de projet et de deux techniciens qui coordonne actuellement le projet. Les ateneus de fabricació partagent des dimensions communes avec les autres AFN (mise à disposition des mêmes outils, machines et logiciels ainsi qu'adhésion aux valeurs de la culture libre). Cependant, les ateneus de fabricació s'en différencient sur plusieurs points, tout particulièrement à ce qui touche leur mission de service public.

1) À la différence des autres ateliers, les ateneus offrent gratuitement leurs services. Ici, la gratuité exprime la possibilité pour un individu d'utiliser l'ensemble des ressources mises à sa disposition sans payer un droit d'accès ou d'utilisation des machines. Seul l'achat des matériaux est à sa charge, dans le cas où il ne souhaite pas utiliser les matériaux de recyclage mis à sa disposition.

2) À long terme, l'objectif est d'ouvrir un ateneu de fabricació dans les 10 districts de la ville : un moyen de couvrir l'ensemble du territoire de Barcelone afin d'assurer un service de proximité. Il existe actuellement quatre ateneus dans les districts de Les Corts, Ciutat Vella et Nou Barris (voir carte 1). La localisation et l'architecture des ateliers facilitent leur visibilité et leur accessibilité.

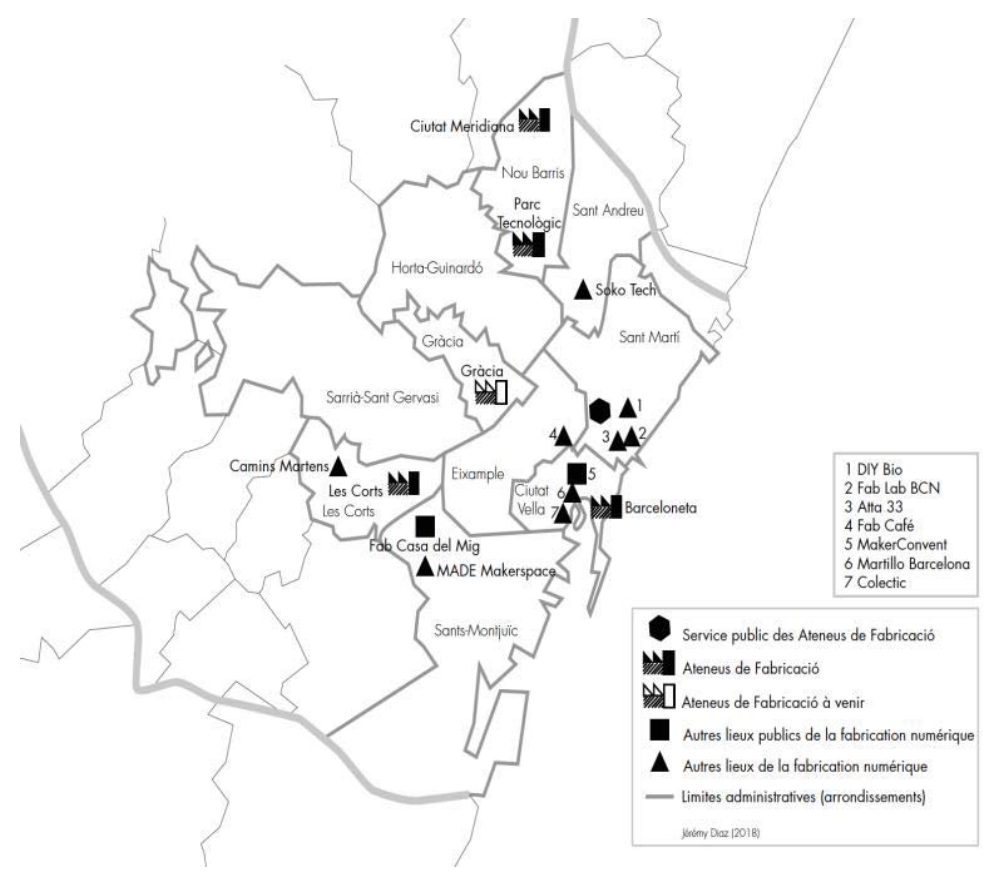

Carte 1 - Les AFN à Barcelone Source : Diaz, 2018 
3) Chaque atelier fonctionne sur le modèle de la contreprestation, appréhendée comme un engagement citoyen envers la collectivité. Tout usager peut utiliser gratuitement les ressources matérielles et immatérielles de l'atelier en échange de la réalisation d'un objet, d'un projet ou d'une activité. Traditionnellement, le fonctionnement des espaces culturels comme les bibliothèques ou les centres communautaires repose sur quatre volets : a) un espace de rencontre où les citoyens peuvent se regrouper afin de partager une activité ou développer un projet commun; b) un espace de consommation culturelle qui permet, par exemple, la consultation de livres, l'utilisation d'ordinateurs ou le prêt de jeux; c) un espace de formation et d'information sous la forme d'ateliers ou d'activités; et d) un espace de diffusion culturelle, par exemple des expositions ou des conférences. La prestation du service est alors garantie par une équipe qui dispose d'un budget pour en assurer le fonctionnement. Cependant, le projet des ateneus de fabricació ne dispose actuellement pas de dota- tions financières suffisantes afin d'assurer pleinement les troisième et quatrième volets. Pour combler ce manque de ressources humaines et financières, la philosophie de gestion des ateneus repose sur la mise en œuvre d'un large système de prestations réciproques pour aider au développement de projets portés par des collectifs, des individus, des institutions ou des entreprises.

4) Chaque ateneu possède une spécificité territoriale en lien avec la nature de l'organisation gérante et les politiques locales du quartier (barrio) dans lequel il se trouve: l'environnement à Barceloneta (voir photo 4), l'emploi à Ciutat Meridiana (voir photo 5), l'inclusion sociale à Les Corts (voir photo 6) et l'entrepreneuriat à Nou Barris (voir photo 7). Cette différenciation thématique vise à favoriser un ancrage territorial avec les acteurs présents sur le territoire en leur donnant la possibilité d'explorer et de prototyper des projets collectifs répondant à des besoins locaux.

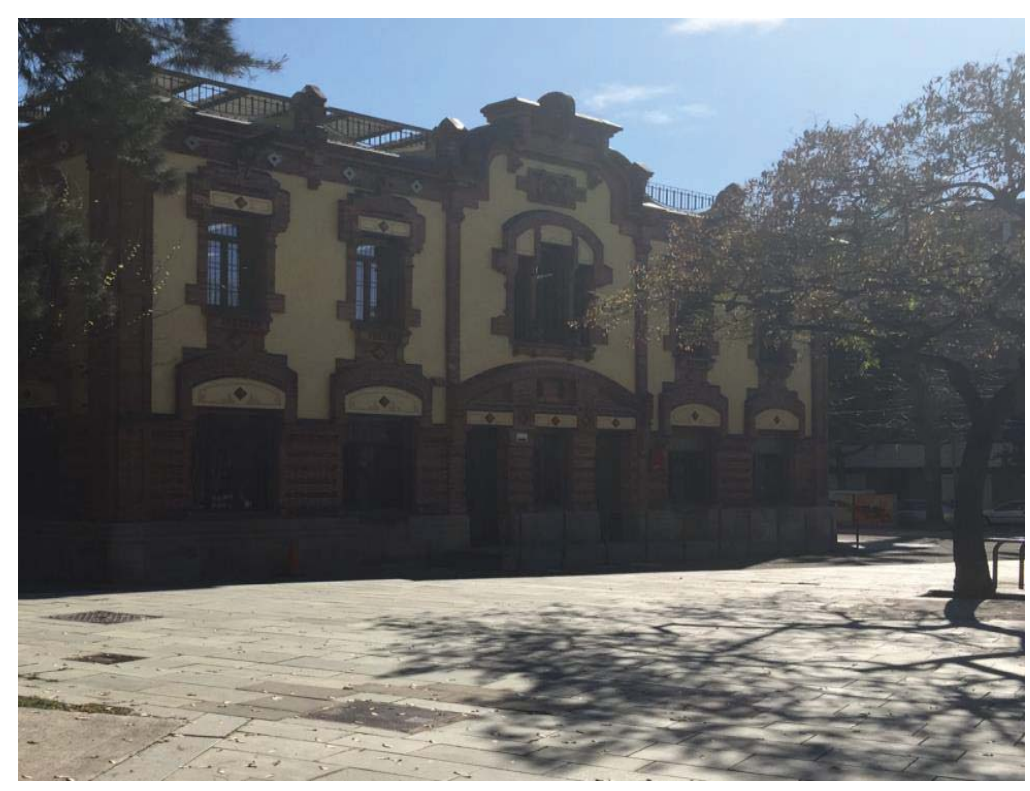

Photo 4-L'environnement à Barceloneta

Source : Diaz et Lefebvre, 2019 


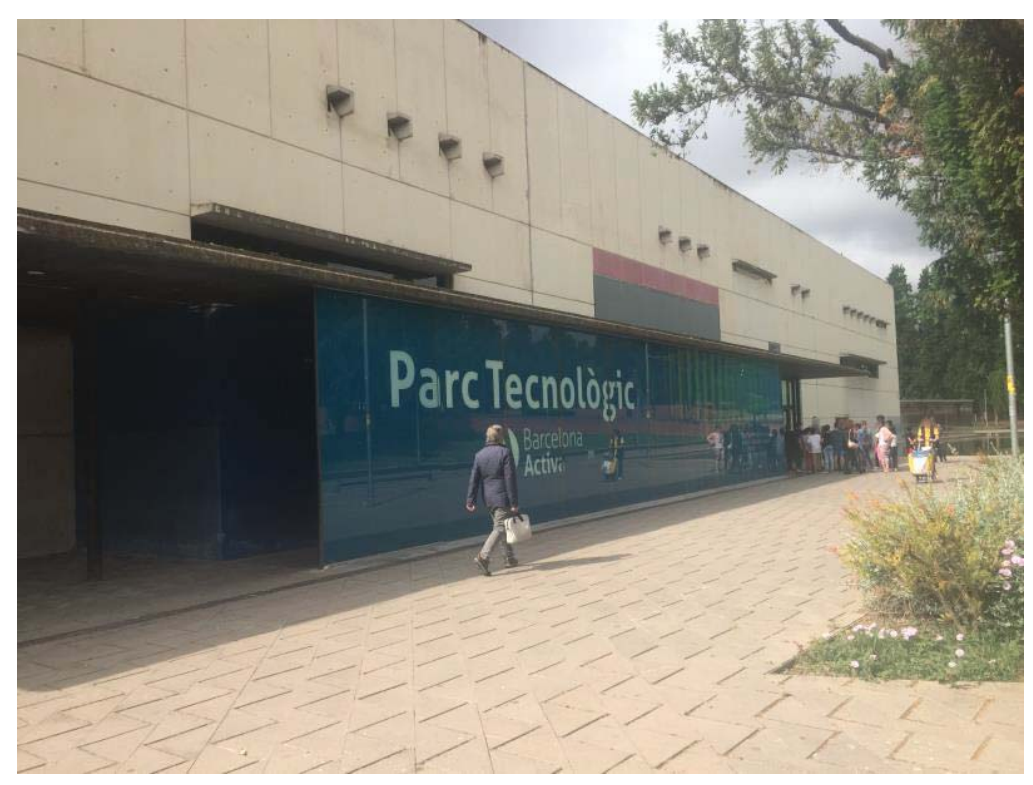

Photo 5 - L'emploi à Ciutat Meridiana

Source : Diaz et Lefebvre, 2019

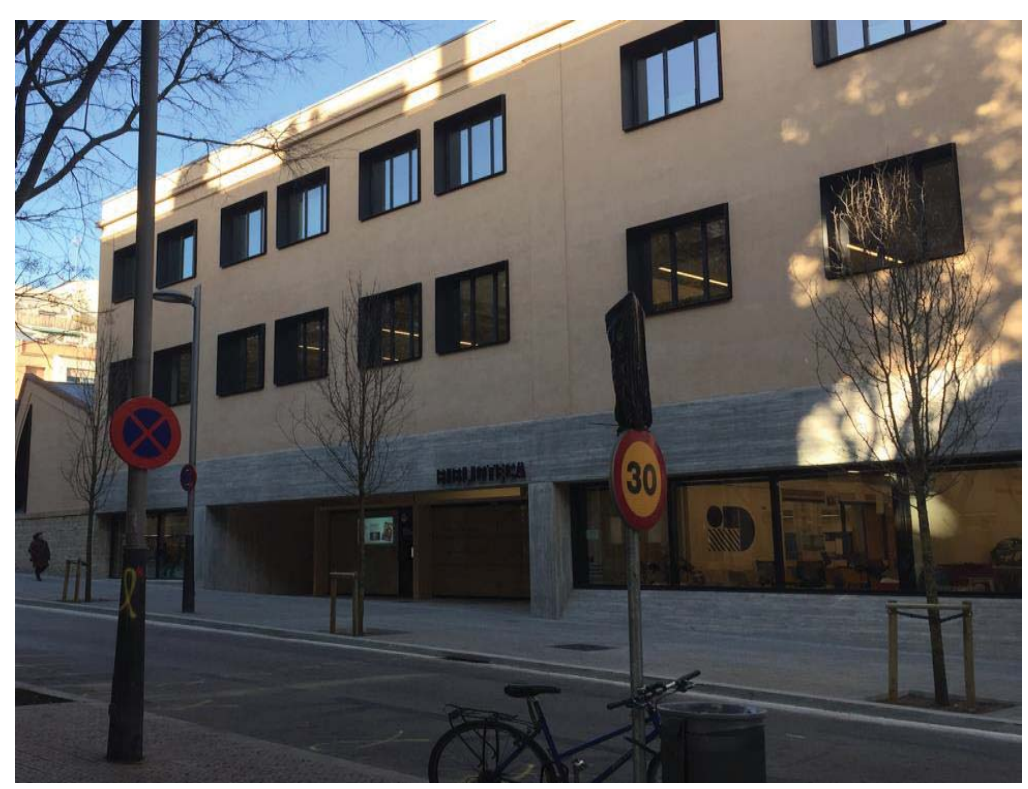

Photo 6 - L'inclusion sociale à Les Corts

Source : Diaz et Lefebvre, 2019 


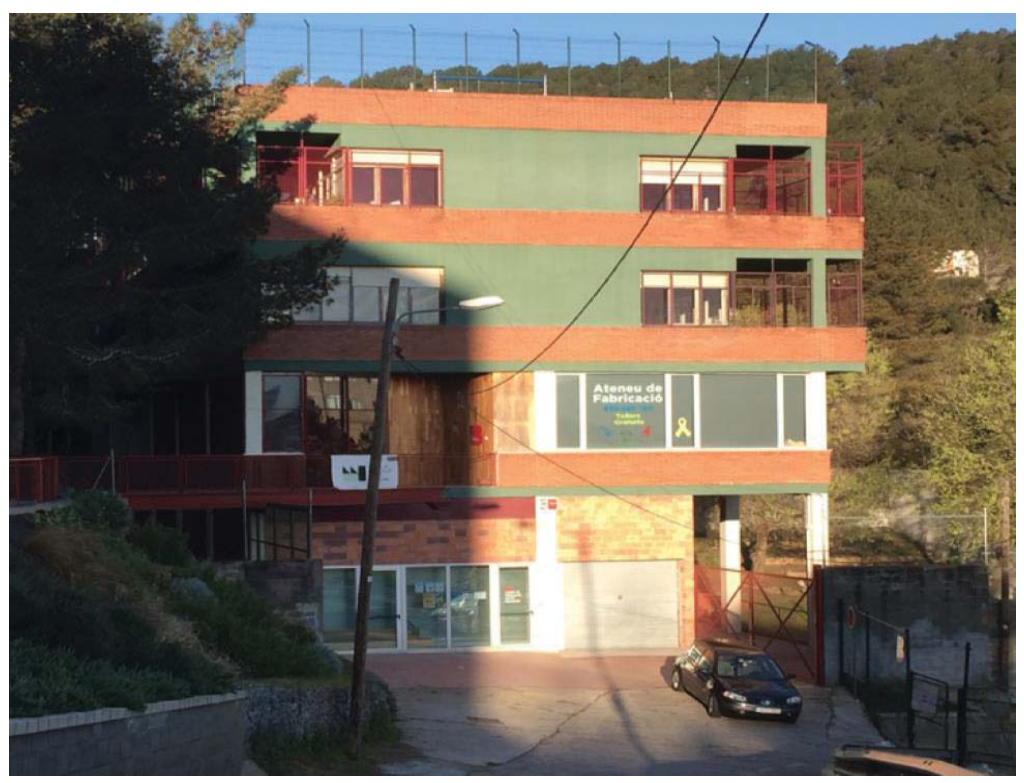

Photo 7 - L'entrepreneuriat à Nou Barris Source : Diaz et Lefebvre, 2019

\subsection{Les programmes, activités et usagers}

Le projet des ateneus de fabricació est encore dans une phase pilote. Cependant, un premier bilan sommaire des activités peut être dressé. De 2015 à 2017, le réseau des ateneus comptabilise un total de 20868 usagers. Les activités sont articulées autour de trois programmes majeurs.

1) Le premier est un programme pédagogique représentant $41 \%$ des usagers totaux. De 2015 à 2017, 452 centres éducatifs ont sollicité ce programme. En collaboration avec la Ville de Barcelona et le Consorci d'Educació de Barcelone, le programme s'adresse à la communauté éducative de la ville (étudiants, enseignants et centres éducatifs de l'enseignement primaire, secondaire et professionnel). Ces activités peuvent être des visites, des ateliers de design et de fabrication, du soutien et de l'accompagnement de projet ou de recherche, des formations ou la création de contenus didactiques.
2) Le second est un programme familial représentant $20 \%$ des usagers totaux. Il propose des activités pédagogiques accessibles au grand public autour de la fabrication numérique (ateliers de robotique, fabrication d'un sac ou de tee-shirt, etc.). Son principal objectif est de réunir trois générations des membres d'une même famille (enfants, parents, grands-parents) autour d'activités techniques afin de renforcer la relation intergénérationnelle et l'engagement des parents dans l'éducation de leurs enfants.

3) Enfin, le troisième programme touche l'innovation sociale et représente $39 \%$ des usagers totaux. De 2015 à 2017, 4738 activités ont été réalisées auprès des usagers. Le programme vise essentiellement à soutenir tout projet de personnes, de collectifs, d'institutions et d'entreprises souhaitant connaitre, apprendre et fabriquer un objet à l'aide des outils de la fabrication numérique (voir photo 8). 


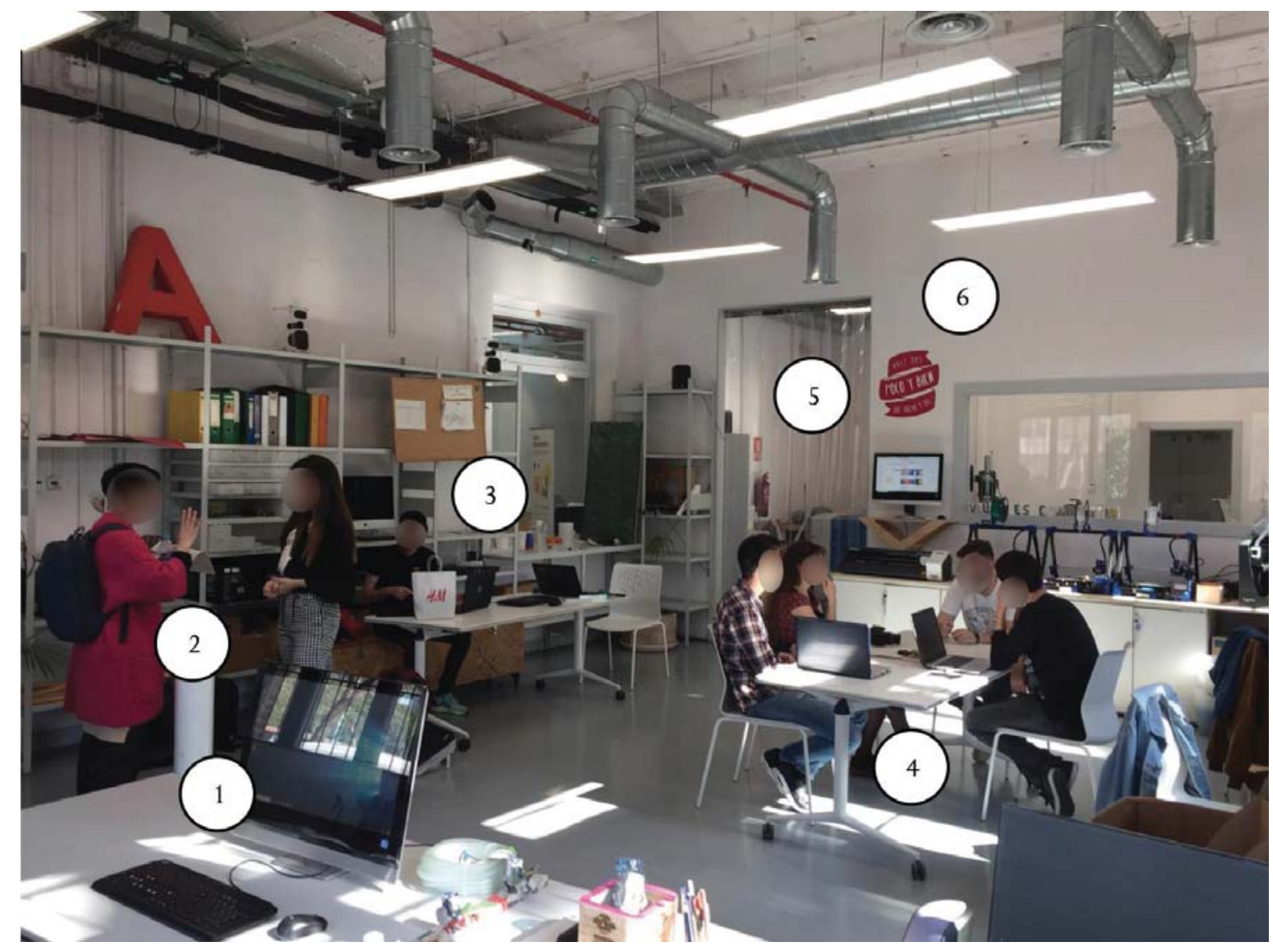

Photo 8- Parcours de l'usager au sein de l'ateneu de fabricació de Barceloneta tel qu'il a été pensé par les dirigeants du réseau des ateneus de fabricació Source : Diaz et Lefebvre, 2019

Légende de la photo :

1) Le recrutement des usagers s'opère de trois façons : la participation à un programme développé par l'ateneu, l'accueil physique au sein de l'édifice ou la soumission électronique du projet sur le site Internet.

2) En théorie, chaque projet est évalué à la lumière de trois critères précis : viabilité technique, économique et sociale.

3) Une fois le projet sélectionné, le personnel de l'atenen invite l'usager à définir le problème éprouvé - la plupart du temps dans la vie quotidienne - et de déterminer les contraintes techniques et budgétaires auxquelles il fait face.

4) Le personnel de l'ateneu peut orienter l'usager vers des solutions techniques existantes ou bien l'accompagner vers l'élaboration d'une nouvelle solution technique.

5) Une fois en possession du fichier qui comporte la modélisation (en 2D ou 3D) de son objet, l'usager procède à l'étape du prototypage. À cette étape, le fichier créé peut être partagé, diffusé ou téléchargé par d'autres par le biais des plateformes numériques à l'interne ou à l'externe. L'usager doit bien saisir le fonctionnement de la machine à commande numérique qui nécessite le réglage de différents paramètres en fonction des matériaux utilisés. Une fois toutes ces étapes effectuées, l'usager est enfin en possession d'un prototype.

6) À la fin du projet, l'usager doit réaliser une contreprestation en accord avec le personnel de l'ateneu de fabricació. 
En raison de l'état actuel des données disponibles, il est difficile de tirer des conclusions définitives de ces premières années d'activité à Barcelone. Le nombre important d'usagers accueillis démontre que les ateneus de fabricació ont permis de rendre la fabrication numérique plus accessible au grand public. Cependant, plusieurs interrogations subsistent quant à l'assimilation réelle de savoir-faire techniques par les citoyens.

\section{Conclusion}

Cette étude exploratoire préfigure une analyse plus systématique dans le cadre d'une thèse en études urbaines en cours par l'un des auteurs. Plusieurs chercheurs ont exploré les relations entre l'éducation et le territoire en montrant les effets positifs d'une diversité de dispositifs et lieux à l'extérieur des institutions scolaires sur l'éducation, la formation et le développement local (Dussaux, 2010; Bier, Chambon et de Queiroz, 2010; Ben Ayed, 2018). Pour contribuer à ce débat autour des « territoires apprenants » (Bier, 2010), nous avons montré la façon dont plusieurs villes tentent de valoriser l'expérimentation et l'acquisition de savoir-faire techniques dans le sillage du succès international du mouvement maker.

Depuis 40 ans, plusieurs initiatives autour de la diffusion d'une culture scientifique et technique se sont développées dans les pays occidentaux (Deforge, 1993; Paquette, 2011). Cette tendance s'est accentuée dans le cadre de l'économie de la connaissance. Cependant, plusieurs auteurs constatent que les initiatives n'ont jamais permis de véri- tablement régler les écarts sociaux dans l'appropriation des technologies (Godin, 1999; Las Vergnas, 2017). Les nouveaux arrivants du mouvement maker déclarent se distinguer des initiatives traditionnelles en ne se concentrant ni sur la visite d'exposition ni sur l'activité pédagogique, mais d'abord sur le développement individuel de savoirs et de savoir-faire techniques à travers la fabrication d'un objet (Chicoineau, 2017). Ils viendraient ainsi combler un manque d'expérimentation concrète dans les activités de vulgarisation des technologies.

Or, l'appropriation d'une technologie par une société exige la mise en place de dispositifs capables de mettre les outils, machines et procédés techniques à la disposition de l'ensemble de la collectivité. À Barcelone, la mise en œuvre du réseau des ateneus de fabricació semble très prometteuse. Dans un proche avenir, l'enjeu sera de déterminer si ce projet participe à une action culturelle de prestige pour la ville de Barcelone, plutôt que d'encourager une véritable appropriation citoyenne de ces moyens technologiques de fabrication. Car, contrairement à l'une des croyances les plus tenaces concernant la technologie numérique, l'« ouverture » ou la « libération » d'une ressource matérielle ou immatérielle n'entraînent pas nécessairement une collaboration élargie d'acteurs permettant de trouver des applications économiques, sociales ou politiques à ces technologies. Il faut désormais penser les conditions d'appropriation de ces ressources en évaluant leurs réelles utilités pour le développement des territoires dans lesquels s'insèrent les AFN.

\section{NOTES}

1 Le mouvement maker naît aux États-Unis lors du lancement du magazine Make! et de l'organisation des premiers festivals Maker Faire à San Francisco et à New York en 2005. Ce mouvement est d'abord une entreprise commerciale consacrée à la commercialisation de biens et loisirs du bricolage et de la culture Do it yourself (DIY) tournée vers la technologie. À mesure que les ventes et les événements se multiplient dans les métropoles mondiales, le terme s'impose dans le langage courant pour désigner la convergence d'acteurs et de pratiques issus de la culture numérique et de l'artisanat plus traditionnels.

2 Entre autres, les caractéristiques de ces ateliers comprennent : 1) l'inventaire des outils et machines disponibles; 2) le statut juridique, la mission et les objectifs de l'organisation; 3) la situation géographique (localisation, environnement du local, surface occupée, capacité d'accueil); 4) le modèle économique et l'offre de service; 5) l'équipe encadrante (nombre et profils des salariés, présence de bénévoles); 6) les sources de financement et la relation aux pouvoirs publics; 7) les pratiques des usagers (types d'activités, comportements, sociabilité); 8) les types de projets; 9) les modalités d'échange des connaissances (formation des visiteurs, documentation des œuvres, échanges de connaissances tacites, activités pédagogiques); et 10) l'insertion dans des réseaux internationaux. 


\section{RÉFÉRENCES}

Ajuntament de Barcelona. (2016). Libre blau dels Ateneus de Fabricació. Barcelone, Espagne.

APUR. (2016). L’innovation à Paris et dans la Métropole du Grand Paris : données sur les startups, les lieux, les méthodes (Rapport). Repéré à www.apur.org/fr/nos-travaux/innovation-paris-metropole-grand-paris-donnees-startups-lieuxmethodes

Barrett, T., Pizzico, M., Levy, B., Nagel, R., Linsey, J., Talley, K. ... Newstetter, W. (2015, juin). A review of university maker spaces. Communication présentée à la $122^{\mathrm{e}}$ Conférence annuelle et exposition de l'American Society Engineering Education, Seattle, WA. Repéré à https://smartech.gatech. edu/handle/1853/53813

Ben Ayed, C. (2018). Éducation et territoire : retour sur un objet sociologique mal ajusté. Sciences de l'éducation - Pour l'ère nouvelle, 51(1), 15-35. doi: 10.3917/1sdle.511.0015

Bier, B. (2010). «Territoire apprenant » : les enjeux d'une définition. Spécificités, 3(1), 7-18. doi: 10.3917/spec.003.0007

Bier, B., Chambon, A. et de Queiroz, J.-M. (2010). Mutations territoriales et éducation : de la forme scolaire vers la forme éducative, Paris, France : ESF.

Blikstein, P. (2013). Digital fabrication and «making» in education: The democratization of invention. Dans J. WalterHerrmann et C. Büching (dir.), FabLabs: Of machines, makers and inventors (p. 1-27). Bielefeld, Allemagne: Transcript-Verlag. doi: $10.14361 /$ transcript. 9783839423820.203

Bowden, J. (2016). Who makes a makerspace? Makerspace governance in Toronto, Ontario, and London, Ontario (Thèse de doctorat). Université McGill, Montréal, QC. Repéré à http://digitool.Library.McGill.CA:80/R/-?func=dbin-jumpfulletobject_id=142962etsilo_library=GEN01

Brahms, L. et Crowley, K. (2016). Learning to make in the museum: The role of maker educators. Dans P. Pepler, E. Halverson et Y. Kafai (dir.), Makeology in K-12 higher, and informal education: The maker movement and the future of learning (chap. 2). Londres, R.-U.: Routledge. doi: 10.4324/9781315726519-2

Budge, K. (2018). Making in the city: Disjunctures between public discourse and urban policy. Australian Geographer, 50(2), 1-15. doi: 10.1080/00049182.2018.1503045

Calgary Economic Development (CED). (2017). How makerspaces support innovative urban economies (Rapport). Repéré à www.calgaryeconomicdevelopment.com/dmsdocument/120

California Community College. (2016). CCC maker initiative. Repéré à https://cccmaker.com/about/ccc-maker-initiative

Capdevila, I. (2017). A typology of localized spaces of collaborative innovation. Dans M. van Ham, D. Reuschke, R. Kleinhans, C. Mason et S. Syrett (dir.), Entrepreneurial Neighbourhoods (p. 80-97). Cheltenham, R.-U.: Edward Elgar. doi: $10.4337 / 9781785367243.00013$

Chicoineau, L. (2017). La culture scientifique et technique est-elle en train de se faire une place au panthéon de la culture? Nectart, 5(2), 58-65. Repéré à www.cairn.info/revue-nectart-2017-2-p-58.htm

Commissariat général à l'égalité des territoires (CGET). (2018). L'État s'engage pour soutenir et accélérer la dynamique des tiers lieux dans les territoires. Paris, France : Ministère de la Cohésion des territoires et des Relations avec les collectivités territoriales. Repéré à www.cget.gouv.fr/actualites/l-etat-s-engage-pour-soutenir-et-accelerer-la-dynamique-des-tiers-lieuxdans-les-territoires

Deforge, Y. (1993). De l'education technologique à la culture technique : pour une maîtrise sociale de la technique. Paris, France : ESF.

Demoustier, D. et Rousselière, D. (2005). L'économie sociale et coopérative des associationnistes : de la critique des fondements de l'économie politique à la régulation sociale du marché. Annals of Public and Cooperative Economics, 76(4), 517-543. doi: 10.1111/j.1370-4788.2005.00288.x

Diaz, J., Tomàs, M. et Lefebvre, S. (2018, juin). « La construcción social de la ciudad digital : el caso de los ateneus de fabricació ». Communication présentée à la 14e International Conference on Internet, Law et Politics (IDP) de l'UOC, Barcelone, Espagne.

Diez, T. (2018). Fab City: The mass distribution of (almost) everything. Repéré à https://issuu.com/iaac/docs/fabcitymassdistribution

Dussaux, M. (2010). L'éducation au développement durable : de la ville éducatrice au territoire apprenant? Spécificités, 3(1), 39-56. doi: 10.3917/spec.003.0039 
Eychenne, F. (2012). Fab lab : l'avant-garde de la nouvelle révolution industrielle. Limoges, France : FYP.

Ferchaud, F. (2017). Les communs urbains à l'épreuve du terrain : le cas des lieux de fabrication numérique. Netcom : réseanx, communication et territoires, $31(1 / 2)$, 53-76. doi: 10.4000/netcom.2628

Fourmond, T. et Gastall, U. (2014). DIY, makers, fablabs : à la recherche de l'autonomie. Repéré à www.ritimo.org/DIYmakers-fablabs-a-la-recherche-de-l-autonomie

Godin, B. (1999). Les usages sociaux de la culture scientifique. Sainte-Foy, QC : Presses de l’Université Laval.

Government of the United Kingdom. (2016). Libraries deliver: Ambition for public libraries in England 2016 to 2021 (Rapport). Repéré à www.gov.uk/government/publications/libraries-deliver-ambition-for-public-libraries-in-england2016-to-2021

Hagel, J., Brown, J. S. et Kulasooriya, D. (2014). A movement in the making. New York, NY: Deloitte Consulting. Repéré à www2.deloitte.com/insights/us/en/topics/emerging-technologies/a-movement-in-the-making.html

Halverson, E. R. et Sheridan, K. (2014). The maker movement in education. Harvard Educational Review, 84(4), 495-504. doi: 10.17763/haer.84.4.34j1g68140382063

Harris, J. et Cooper, C. (2015). Make room for a makerspace. Computers in Libraries, 35(2), 5-9.

Hirshberg, P., Dougherty, D. et Kadanoff, M. (2016). Maker City: A practical guide to reinvention in our cities. San Francisco, CA: Maker Media. Repéré à https://proquest.safaribooksonline.com/9781680452907

Kelly, T. (1952). The origin of mechanics' institutes. British Journal of Educational Studies, 1(1), 17-27. doi: 10.2307/3119430

Klein, J.-L. (2016). Le leadership : un facteur vital pour le développement territorial. Dans É. Glon et B. Pecqueur (dir.), $A u$ cour des territoires créatifs : proximités et ressources territoriales (p. 49-57). Rennes, France : Presses universitaires de Rennes.

Klein, J.-L. et Pecqueur, B. (2017). Living Labs, innovation sociale et territoire. Revue canadienne des sciences régionales, 40(1), 1-4. Repéré à www.cjrs-rcsr.org/V40/cjrsrcsr40-1-0Klein-pecqueur.pdf

Klein, J.-L., Soussi, S. A., Koop, K. et Pecqueur, B. (dir.). (2016). L'innovation locale à l'épreuve du global : un défi pour les acteurs. Québec, QC : Presses de l'Université du Québec.

Lægran, A. S. et Stewart, J. (2003). Nerdy, trendy or healthy? Configuring the Internet café. New Media \& Society, 5(3), 357-377. doi: $10.1177 / 14614448030053004$

Lallement, M. (2015). L'âge du faire : backing, travail, anarcbie. Paris, France : Seuil.

Las Vergnas, O. (2017). Répétitions des discours sur la culture scientifique et technique et effets de la catégorisation scientifique scolaire. Innovations, 52(1), 85-109. doi: 10.3917/inno.052.0085

Levy, S. (2010). Hackers: Heroes of the computer revolution. Sebastopol, CA: O’Reilly Media.

Liefooghe, C. (2017). Tiers-lieux, coworking spaces et FabLabs : nouveaux lieux, nouveaux liens et construction de communautés de connaissance créatives. Dans D. Mons et D. Paris (dir.), Lille, métropole créative? : nouveaux liens, nouveaux lieux, nouveaux territoires (p. 183-222). Lille, France : Septentrion. Repéré à http://books.openedition.org/septentrion/13446

Liefooghe, C. (2018). Les tiers-lieux à l'ère du numérique : diffusion spatiale d’une utopie socio-économique. Géographie, économie, société, 20(1), 33-61. doi: 10.3166/ges.20.2017.0028

Lô, A. (2017). Un FabLab d'entreprise pour favoriser l'ambidextrie des salariés. Revue française de gestion, 43(264), 81-99. doi: $10.3166 / \mathrm{rfg} .2017 .00113$

Mariotti, I., Pacchi, C. et Di Vita, S. (2017). Co-working spaces in Milan: Location patterns and urban effects. Journal of Urban Technology, 24(3), 47-66. doi: 10.1080/10630732.2017.1311556

National League of Cities. (2016). How cities can grow the maker movement (Rapport). Repéré à www.nlc.org/resource/how-citiescan-grow-the-maker-movement-report

Niaros, V., Kostakis, V. et Drechsler, W. (2017). Making (in) the smart city: The emergence of makerspaces. Telematics and Informatics, 34(1), 1143-1152. doi: 10.1016/j.tele.2017.05.004 
NYC Planning. (2018). Can industrial mixed-use buildings work in NYC? (Rapport). Repéré à www1.nyc.gov/assets/ planning/download/pdf/about/dcp-priorities/data-expertise/can-industrial-mixed-use-buildings-work-in-nyc.pdf

Pacchi, C. (2017). Sharing economy: Makerspaces, co-working spaces, hybrid workplaces, and new social practices. Dans S. Armondi et S. Di Vita (dir.), Milan: Productions, spatial patterns and urban change (chap. 5). Londres, R.-U.: Routledge.

Paquette, J. (2011). Science as culture and leisure: Cultural policy, industry and scientific culture in the Canadian context. International Journal of Cultural Policy, 17(1), 39-51. doi: 10.1080/10286630903531356

Pecqueur, B. (2016). Le développement territorial : une voie de sortie de crise pour les collectivités locales. Dans J.-L. Klein, S. A. Soussi, K. Koop et B. Pecqueur (dir.), L'innovation locale à l'éprewve du global : un défi pour les acteurs (p. 15-25). Québec, QC : Presses de l’Université du Québec.

Pratt Center (2016). Creating an innovative mix: What we can learn from San Francisco. Repéré à https://prattcenter.net/sites/ default/files/25_kent_policy_brief_final_0.pdf

Rosa, P., Ferretti, F., Panella, F. et Wanner, M. (2017). Overview of the maker movement in the European Union. Luxembourg: Publications Office of the European Union.

Sheridan, K., Halverson, E. R., Litts, B., Brahms, L., Jacobs-Priebe, L. et Owens, T. (2014). Learning in the making: A comparative case study of three makerspaces. Harvard Educational Review, 84(4), 505-531. doi: 10.17763/haer.84.4.brr34733723j648u

Smart Growth America. (2017). Made in place: Small-scale manufacturing and neighborbood revitalization. Repéré à https://smartgrowthamerica.org/resources/made-in-place

Smith, A., Hielscher, S., Dickel, S., Soderberg, J. et van Oost, E. (2013). Grassroots digital fabrication and makerspaces: Reconfiguring, relocating and recalibrating innovation? (SSRN Scholarly Paper No. ID 2731835). Repéré à https://papers.ssrn.com/ abstract $=2731835$

Suire, R. (2016). La performance des lieux de cocréation de connaissances. Réseaux, 196(2), 81-109. doi: 10.3917/res.196.0081

Torre, A. (2015). Théorie du développement territorial. Géographie, économie, société, 17(3), 273-288. doi: 10.3166/ges.17.273-288

Urban Redevelopment Authority of Pittsburgh (URAP). (2015). Roadmap for inclusive innovation (Rapport). Repéré à http://pittsburghpa.gov/innovation-performance/innovationroadmap/documents/Pittsburgh-Roadmap-forInclusive-Innovation.pdf

Van Holm, E. (2015). Makerspaces: Contributions to economic development in tier 2 and smaller cities. Atlanta, GA: Georgia Institute of Technology and Georgia State University. Repéré à http://stip.gatech.edu/wp-content/uploads/2015/08/Van-HolmMakerspaces-Project-Report.pdf

Ville d'Ottawa (2018). Zoning study: Small-scale industrial uses in commercial zones. Ottawa, ON: Planning, Infrastructure and Economic Development Department. Repéré à https://ottawa.ca/en/city-hall/public-engagement/projects/zoningstudy-small-scale-industrial-uses-commercial-zones

Wen, W. (2017). Making in China: Is maker culture changing China's creative landscape? International Journal of Cultural Studies, 20(4), 343-360. doi: 10.1177/1367877917705154

Wolf-Powers, L., Doussard, M., Schrock, G., Heying, C., Eisenburger, M. et Marotta, S. (2017). The maker movement and urban economic development. Journal of the American Planning Association, 83(4), 365-376. doi: 10.1080/01944363.2017.1360787

Wong, A. et Partridge, H. (2016). Making as learning: Makerspaces in universities. Australian Academic \& Research Libraries, 47(3), 143-159. doi: 10.1080/00048623.2016.1228163

Wusten, H. van der. (2016). La ville fonctionnelle et les modèles urbains qui lui ont succédé : exemples d'une pratique politique en train de se globaliser. EchoGéo, 36. doi : 10.4000/echogeo.14634 